\title{
NOUVELLE
}

\section{Prévenir la progression de la pathologie Tau par immunothérapie passive}

Laurence Ozmen, Ludovic Collin
F. Hoffmann-La Roche Ltd.,

Preclinical Research Neuroscience, PCDMR, Grenzacherstrasse, CH-4070 Basel,

Suisse.

ludovic.collin@roche.com
La protéine Tau et les maladies neurodégénératives

Les maladies neurodégénératives regroupent un ensemble de pathologies touchant le système nerveux. Les plus connues sont la maladie d'Alzheimer et la maladie de Parkinson, pour lesquelles la prévalence augmente avec l'âge [1]. L'incidence de ces maladies ne cessera d'augmenter au cours des prochaines décennies.

Les maladies neurodégénératives sont caractérisées par une détérioration progressive des neurones entraînant un dysfonctionnement du système nerveux et une perte graduelle des capacités cognitives et/ou motrices. Une des caractéristiques histopathologiques de ces maladies est l'accumulation d'agrégats protéiques intra- ou extracellulaires qui précède la dégénérescence neuronale. Certains de ces agrégats sont spécifiques d'une pathologie, comme les plaques amyloïdes révélatrices de la maladie d'Alzheimer. D'autres, comme ceux formés par la protéine Tau, sont communs à plusieurs pathologies. Tau forme des agrégats intracellulaires qui, dans leur forme ultime, sont nommés enchevêtrements neurofibrillaires (tangles ou NFT neurofibrillary tangles). On les retrouve dans la maladie d'Alzheimer, mais aussi dans de nombreuses autres maladies neurodégénératives regroupées sous la dénomination de tauopathies (démence fronto-temporale, dégénération corticobasale, maladie de Pick, etc.). Un lien direct entre l'agrégation de la protéine Tau et la neurodégénérescence a été établi avec la découverte de mutations de Tau causatives d'une démence fami- liale (FTDP-17) [2]. Des mutations de la protéine Tau ne sont pas directement associées à la maladie d'Alzheimer, mais les NFT sont une des signatures pathologiques de cette maladie et leur présence corrèle avec la sévérité de la démence $[1,3]$. Braak et al. ont montré que la progression de la pathologie Tau suit un circuit de neurones interconnectés qui est défini et prévisible. Chez les patients atteints de la maladie d'Alzheimer, les enchevêtrements neurofibrillaires sont initialement détectés dans le cortex transentorhinal, la pathologie progresse ensuite au cortex enthorinal (région proche de l'hippocampe, qui intègre des informations d'origines diverses avant qu'elles ne rejoignent l'hippocampe) en suivant les projections glutamatergiques, puis à l'hippocampe, à l'amygdale et au néocortex $[1,4]$. Le mécanisme exact de cette progression n'est pas élucidé, mais des expérimentations précliniques suggèrent qu'il pourrait être similaire à celui de la propagation de la protéine prion par transmission de cellule à cellule [5].

\section{La protéine Tau : de la physiologie} à la pathologie

Tau est une protéine monomérique soluble spécifiquement présente dans les axones des neurones où elle est associée aux microtubules et régule leur dynamique. Des modifications post-traductionnelles, telles que phosphorylation ou acétylation, régulent ses fonctions intracellulaires. En effet, Tau possède de nombreux résidus sérine et thréonine susceptibles d'être phosphorylés (plus de 40 sites identifiés). Dans des conditions pathologiques, Tau devient hyperphosphorylée: des sites additionnels aux sites physiologiques sont phosphorylés, et la fonction de la protéine s'en trouve modifiée. Tau n'interagit plus avec les microtubules de l'axone, mais diffuse dans le soma puis les dendrites neuronales où elle s'accumule. Cela favorise son agrégation et la formation d'oligomères, puis d'agrégats plus structurés: les enchevêtrements neurofibrillaires [6]. La phosphorylation de Tau se fait séquentiellement : certains sites sont phosphorylés précocement (Ser202, Ser235, Ser422), d'autres le sont à des stades plus avancés de la maladie (Ser396/404) [7]. Cette phosphorylation séquentielle est corrélée au degré de cytopathologie neuronale et à la formation des NFT : les oligomères forment tout d'abord des agrégats diffus ou sphériques dans le soma des neurones (pré-NFT). Les cellules contenant ce type d'agrégat ont une morphologie tout à fait normale et présentent de nombreuses dendrites. L'accumulation progressive de pré-NFT induit la formation de NFT dans le cytoplasme neuronal (iNFT) où le noyau cellulaire est présent, mais les dendrites sont détériorés. Finalement, on aboutit à une cellule fantôme : les NFT extraneuronaux (eNFT), qui consistent en des agrégats filamenteux extracellulaires ayant gardé la forme d'une cellule neuronale mais sans noyau cellulaire détectable (cellule fantôme) [6]. Les NFT ont longtemps été considérés comme la forme toxique de Tau, car ils constituent le stade final d'un processus pathologique [8]. Cependant, on pense que les formes 
oligomériques sont responsables de la transmission intercellulaire de la pathologie, et de ce fait, pourraient être les plus toxiques. En effet, pendant longtemps, Tau a été considérée comme une protéine exclusivement intracellulaire. Toutefois, la sécrétion de la protéine Tau native a récemment été décrite [9], observation en faveur du concept selon lequel Tau pourrait être fonctionnelle hors du cytoplasme et transmettre la pathologie de cellule à cellule.

\section{Interventions thérapeutiques}

Nos connaissances actuelles suggèrent que la neurodégénérescence induite par la molécule Tau résulte à la fois d'une perte de fonction (altérations de la fonction des microtubules et du transport axonal) et d'un gain de fonction toxique (agrégats de protéine Tau hyper-phosphorylée). De ce fait, différentes approches thérapeutiques peuvent être envisagées.

\section{Réduction des taux de protéine Tau}

Le taux d'une protéine intracellulaire est la résultante de la synthèse et de la dégradation de cette protéine. Les mécanismes moléculaires contrôlant la synthèse de la protéine Tau sont mal connus, cette approche reste donc pour le moment au stade exploratoire. Deux systèmes protéolytiques sont responsables de la dégradation des protéines dans les neurones: le protéasome et la voie d'autophagie impliquant la dégradation lysosomale. Ces voies, ainsi que les molécules qui les contrôlent, sont ubiquitaires et ont des effets pléiotropes. Moduler leur fonction et/ou leur expression est attractif, mais pourrait s'avérer toxique.

\section{Inhibition de l'agrégation de Tau}

Une relation de cause à effet entre l'hyperphosphorylation de Tau et son agrégation n'a pas été démontrée, mais leur forte corrélation encourage la recherche d'inhibiteurs de protéines kinases. De nombreuses kinases peuvent phosphoryler Tau (GSK3b, CDK5, MAPK etc.), mais aucune ne lui est spécifique, rendant cette approche risquée [10]. Une alternative à l'inhibition de kinases est l'activation de la phosphatase PP2A. Comme précédemment, la multitude de substrats et la complexité de la phosphatase rendent l'approche thérapeutique difficile.

Il a également été montré que l'acétylation de Tau inhibe fortement son agrégation. Néanmoins, des résultats thérapeutiques contradictoires ont été publiés avec des inhibiteurs de HDAC6, un modulateur non spécifique de l'acétylation de Tau [11].

Une autre hypothèse incriminée pour expliquer la formation des NFT est la fragmentation de Tau. L'apparition de fragments protéiques est observée avant le phénomène d'agrégation [12]. L'inhibition du ou des enzymes responsable(s) du clivage de la protéine (caspases ou autres) pourrait se révéler efficace.

Finalement, de nombreux inhibiteurs de l'agrégation de Tau ont été identifiés in vitro, mais la démonstration de leur efficacité in vivo reste limitée [13]. Inhiber l'agrégation de Tau peut avoir des conséquences néfastes s'il en résulte une augmentation des oligomères solubles. Une solution serait d'associer cette approche thérapeutique avec une deuxième classe de molécules capable de promouvoir la dégradation des oligomères.

\section{L'immunothérapie dirigée contre Tau}

L'immunothérapie est une approche thérapeutique envisagée dans différentes maladies neurodégénératives; son but est d'éliminer préférentiellement les molécules qui affectent la viabilité neuronale à l'aide d'anticorps spécifiques. Cette approche est d'autant plus intéressante si l'on considère la présence de la protéine Tau dans l'espace extracellulaire et la transmission de la pathologie de cellule à cellule. Bloquer cette transmission pourrait stopper la propagation de la pathologie.

Des résultats encourageants ont été obtenus dans la majorité des études d'immunothérapie visant Tau, mais de nombreuses questions restent sans réponse quant aux mécanismes d'action à l'œuvre. Certaines études suggèrent que les anticorps ne pénètrent pas dans les cellules et agissent dans le compartiment extracellulaire. C'est par exemple le cas d'anticorps spécifiques (Ig totales) des formes oligomériques ou capables d'inhiber l'agrégation de Tau [14]. D’autres études précliniques d'immunisations actives chez la souris utilisant comme immunogènes des peptides phosphorylés ont montré que les anticorps produits sont capables d'entrer dans les neurones, de se lier aux NFT et de ralentir la progression de la pathologie [15-17]. Cependant, l'injection répétée de peptides recombinants lors de telles immunisations actives présente des risques [18]. De ce fait, l'immunisation passive (administration directe d'anticorps spécifiques) est préférable. Des résultats prometteurs ont été obtenus dans différents modèles précliniques utilisant des anticorps spécifiques de certains épitopes phosphorylés comme Ser396/404 [16]; ces épitopes ne sont toutefois pas spécifiques de conditions pathologiques.

\section{Exemple de l'anticorps Mab86 dirigé contre Tau phosphorylée} Hoffmann-La Roche a développé l'anticorps Mab86 [19] dirigé contre la protéine Tau spécifiquement phosphorylée sur la sérine 422 (TauS422). Nous venons de montrer, dans un modèle de souris triple transgénique exprimant les protéines humaines APP, préseniline 2 et Tau (TauPS2APP), que Mab86 reconnaît les différentes formes d'agrégats Tau décrites par Augustinack et al. [6] chez des patients atteints de la maladie d'Alzheimer [20]. En effet, Mab86 reconnaît des pré-NFT, des iNFT et des eNFT chez ces animaux. De plus, ces travaux apportent un début d'explication quant au possible mécanisme d'action intracellulaire de l'immunothérapie. Nous avons en effet suivi le cheminement de Mab86 dans le cerveau des souris après administration intraveineuse de l'anticorps. Nous montrons que l'anticorps (1) traverse la barrière encéphalique, et (2) reconnaît de manière spécifique les neurones qui 
contiennent des NFT phosphorylés sur le résidu TauS422. Des données cellulaires et biochimiques suggèrent que la spécificité de reconnaissance de Mab86 pour cette population de neurones est due à la localisation membranaire de TauS422, dans des domaines lipidiques définis. L'interaction entre Mab86 et son épitope au niveau de la membrane induit l'endocytose du complexe formé comme l'indique la détection de Mab86 dans des structures vésiculaires présentes dans le soma et les dendrites des neurones ciblés. II est connu que les structures vésiculaires issues de l'endocytose peuvent être recyclées et/ou dégradées. Nous montrons, par immunomarquage, que Mab86 est aussi détecté dans les lysosomes. Les complexes antigène-anticorps internalisés sont donc délivrés à ces organites, puis y sont dégradés. Enfin, nous avons aussi observé qu'une administration chronique de Mab86 pendant quatre mois réduit la progression de la pathologie Tau d'environ $50 \%$ (quantification biochimique de la protéine et comptage neuronal) chez les souris TauPS2APP. Ces résultats suggèrent qu'outre son rôle protecteur extracellulaire bloquant la propagation inter-neuronale de la pathologie, l'immunothérapie exerce aussi un rôle intracellulaire actif.

Nous apportons également des arguments suggérant que l'accumulation progressive de TaupS422 dans les neurones a un impact direct sur la fonction lysosomale de ces cellules. En effet, le nombre de lysosomes est inversement correlé à la quantité de Tau pathologique. Cependant, la quantité de Tau pathologique corrèle avec l'accumulation de la protéine p62/SDSTMl, un marqueur d'activité lysosomale. Ces données suggèrent que la libération intracellulaire de Mab86 protègerait les lysosomes contre les effets néfastes des NFT TauS422.

\section{Perspectives}

La corrélation entre les symptômes cliniques et la quantité d'agrégats fibrillaires de Tau chez les patients atteints de Tauopathies désigne Tau comme une cible thérapeutique privilégiée. Néanmoins, de nombreuses questions restent en suspens quant à la forme moléculaire neurotoxique de Tau et son possible mode de propagation. Les modèles précliniques actuels seront-ils suffisants pour répondre à ces questions et prédire l'efficacité des molécules thérapeutiques? Seule l'expérimentation nous permettra de progresser dans la compréhension des mécanismes pathologiques induit par Tau. En attendant, nos résultats [20] enrichissent nos connaissances des mécanismes d'action de l'immunothérapie passive dirigée contre Tau, et ouvrent des perspectives thérapeutiques plus larges car ils démontrent, pour la première fois, la possibilité d'une efficacité intra-cellulaire de l'immunothérapie. $\diamond$

Passive Tau immunotherapy for prevention of Tau pathology

progression

\section{LIENS D'INTÉRÊT}

Les auteurs déclarent n'avoir aucun lien d'intérêt concernant les données publiées dans cet article.

\section{RÉFÉRENCES}

1. Braak H, Braak $\varepsilon$. Neuropathological stageing of Alzheimer-related changes. Acta Neuropath 1991 ; 82 : 239-59.

2. Hutton M, Lendon CL, Rizzu P, et al. Association of missense and 5'-splice-site mutations in tau with the inherited dementia FTDP-17. Nature 1998 ; 393 : 702-5.

3. Arriagada PV, Growdon JH, Hedley-Whyte ET, Hyman BT. Neurofibrillary tangles but not senile plaques parallel duration and severity of Alzheimer's disease. Neurology $1992 ; 42: 631-9$.

4. Zhou J, Gennatas ED, Kramer JH, et al. Predicting regional neurodegeneration from the healthy brain functional connectome. Neuron 2012 ; 73 : 1216-27.

5. Frost B, Diamond MI. Prion-like mechanisms in neurodegenerative diseases. Nat Rev Neurosci 2010 ; $11: 55-9$.
6. Augustinack JC, Schneider A, MandelkowE-M, Hymen BT. Specific Tau phosphorylation sites correlate with severity of neuronal cytopathology in Alzheimer's disease. Acta Neuropathol $2002 ; 103: 26-35$.

7. Deters N, Ittner LM, Gotz J. Divergent phosphorylation pattern of Tau in P301L tau transgenic mice. Eur J Neurosci $2008 ; 28$ : 137-47.

8. Brunden KR, Trojanowski JQ, Lee VM. Advances in taufocused drug discovery for Alzheimer's disease and related tauopathies. Nat Rev Drug Discov 2009 ; 8 : 783-93.

9. Pooler AM, Phillips EC, Lau DH, et al. Physiological release of endogenous tau is stimulated by neuronal activity. EMBO Rep 2013 ; 14 : 389-94.

10. Lee VM, Brunden KR, Hutton M, Trojanowski JQ. Developing therapeutic approaches to tau, selected kinases, and related neuronal protein targets. CSH Perspect Med 2011 ; 1 : a006437.

11. Noack M, Leyk J, Richter-Landsberg C. HDAC6 inhibition results in tau acetylation and modulates tau phosphorylation and degradation in oligodendrocytes. Glia 2014 ; 62 : 535-47.

12. Wang YP, Biernat J, Pickhardt M, et al. Stepwise proteolysis liberates tau fragments that nucleate the Alzheimer-like aggregation of full-length tau in a neuronal cell model. Proc Natl Acad Sci USA 2007 ; 104 : 10252-7.

13. Bulic B, Pickhardt M, Mandelkow $\varepsilon$. Progress and developments in tau aggregation inhibitors for Alzheimer disease.J Med Chem 2013 ; 56 : 4135-55.

14. Yanamandra K, Kfoury $N$, Mahan $T E$, et al. Anti-Tau antibodies that block tau aggregate seeding in vitro markedly decrease pathology and improve cognition in vivo. Neuron $2013 ; 80: 402-14$.

15. Asuni AA, Boutajangout A, Quatermain D, Sigurdsson EM. Immunotherapy targeting pathological tau conformers in a tangle mouse model reduces brain pathology with associated functional improvements. J Neurosci 2007 ; 27 : 9115-29.

16. Boutajangout $A$, Ingadottir J, Davies $P$, Sigurdsson $E M$ Passive immunization targeting phospho-tau protein in a mouse model reduces functional decline and clears tau aggregates from the brain. J Neurochem $2011 ; 118: 658-67$

17. Troquier L, Cailleriez R, Burnouf S, et al. Targeting phosphor-Ser422 by active Tau immunotherapy in the THYTau22 mouse model : a suitable therapeutic approach. Curr Alzheimer Res 2012 ; 9 : 397-405.

18. Rozenstein-Tsalovich L, Grigoriadis N, Lourbopoulos $A$, et al. Repeated immunization of mice with phosphorylated-tau peptides causes neuroinflammation. Exp Neurol 2013 ; 248 : 451-6.

19. Grueninger F, Bohrmann B, Czech C, et al. Phosphorylation of Tau at $\$ 422$ is enhanced by Abeta in TauPS2APP triple transgenic mice. Neurobiol Dis $2010 ; 37: 294-306$.

20. Collin L, Bohrmann B, Göpfert U, et al. Neuronal uptake of tau/pS422 antibody and reduced progression of tau pathology in a mouse model of Alzheimer's disease. Brain 2014 ; 137 : 2834-46.

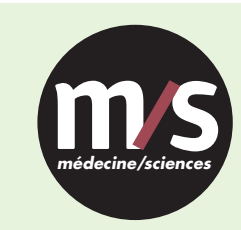

Tarifs d'abonnement $\mathrm{m} / \mathrm{s}-2015$

Abonnez-vous

à médecine/sciences
$>$ Grâce à $m / s$, vivez en direct les progrès des sciences biologiques et médicales

Bulletin d'abonnement page 221 dans ce numéro de $\mathrm{m} / \mathrm{s}$

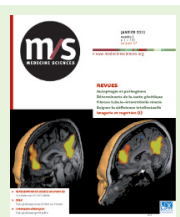

